Copyright $\odot 2008$ Institute of Electrical and Electronics Engineers, Inc.

All rights reserved.

Personal use of this material, including one hard copy reproduction, is permitted.

Permission to reprint, republish and/or distribute this material in whole or in part for any other purposes must be obtained from the IEEE.

For information on obtaining permission, send an e-mail message to stds-ipr@ieee.org.

By choosing to view this document, you agree to all provisions of the copyright laws protecting it.

Individual documents posted on this site may carry slightly different copyright restrictions.

For specific document information, check the copyright notice at the beginning of each document. 


\title{
Combining SOM based Clustering and MGS for Classification of Suspicious Areas within Digital Mammograms
}

\author{
Peter Mc Leod, Brijesh Verma and Rinku Panchal \\ School of Computing Sciences, Central Queensland University \\ North Rockhampton, QLD 4701 Australia \\ peterm@practical.com.au \\ b.verma@cau.edu.au \\ panchal.rinku@gmail.com
}

\begin{abstract}
The fusion of clustering and least square based method for the classification of suspicious areas into benign and malignant classes in digital mammograms was investigated in our previous paper which showed some promising results. This paper extends the investigation by combining a self organising map (SOM) based clustering with modified gramschmidt (MGS) method. The main focus of the research presented in this paper is to investigate the effect that the assignment of input weights from the SOM clustering algorithm have on the efficiency and accuracy of the neural network classifier. A number of experiments have been conducted on a benchmark database. A comparative analysis with our previous results and other known techniques in the literature is presented in this paper.
\end{abstract}

\section{INTRODUCTION}

Malignant cell growth in breast tissues causes breast cancer. The breast tissue composition is identical in both women and men; however women are highly affected by breast cancer compared to men which have an incidence rate of less than $1 \%$. Even though many factors contributing to the risk of women getting breast cancer have been identified, the cause remains unknown. Survival from breast cancer is dependent on the stage it is detected. Early detection, linked to appropriate treatment is currently the most effective strategy to reduce both breast cancer mortality and morbidity increasing the overall survival rate.

Currently mammography is well-recognized as an early detection tool due to its potential to detect breast abnormalities such as masses, calcifications, and other suspicious anomalies in their primitive stages, usually before physical symptoms develop [1-3]. Resultant detected suspicious abnormalities of mammographic screening are further classified as benign or malignant by ultra sound or histopathological examination for advanced treatment options.

The interpretation of mammograms for early stage suspicious abnormalities is a repetitive and fatiguing task with an increasing risk of overlooking abnormalities. It has been estimated that $11-25 \%$ of Breast Cancers are missed during routine screening [4]. Moreover, the classification of detected abnormalities into malignant and benign classes is again a challenging task due to a high resemblance and abundance of variety in search patterns. Currently breast cancer is recognized as the second leading malpractice related condition in clinical practice with a high occurrence of misdiagnosed and delayed treatment cases [5]. Practical studies have illustrated that computer-aided detection systems have efficiently detected early stage breast abnormalities, which were missed by radiologists in first pass mammographic interpretations [6]. Along with skilled radiologists, a computer supported detection and classification technique can effectively improve and accelerate the overall interpretation process.

Since the amount of data that physicians and radiographers must analyse has increased dramatically an accurate and fast diagnostics system is required. Without such a system misdiagnosis can have dire consequences. False negatives can lead to patient demise while false positives are physically and psychologically taxing on patients. In both instances an unnecessary financial and resource burden is placed on our health care system. CAD systems have already demonstrated their ability in improving the diagnostic capabilities of radiographers [7]. Since one of the key features of expertise in a field is skill gained through experience and that feedback can assist in refining and developing skills a CAD system can be an invaluable adjunct in the day to day work and also in the training of radiographers [8]. 
The ability of neural classifiers to learn from the attributes of given class patterns and to classify unknown patterns of given classes into appropriate classes using the acquired knowledge has shown its potential [9-25] in the field of digital mammography. The work conducted by Verma and Panchal [11]; Zhang, Verma and Kumar [13] have shown great potential for finding appropriate features and classifying them into benign and malignant classes. The work conducted by Cheng et al. [9] presents a comparative analysis of various algorithms and techniques for computer-aided detection and classification of microcalcification and mass types of breast abnormalities in digital mammography. Techniques such as artificial neural networks $[10,11,15-18]$, fuzzy logic $[10,26-$ $28]$, and wavelet transforms $[29,30]$ are the most commonly used forms of detection and classification of malignant and benign patterns in digital mammograms. Chitre et al. [17] compared the artificial neural networks and the statistical methods for microcalcification pattern classification. They obtained a classification rate of $60 \%$, which was better than the statistical classifiers. A comparative study of a radial basis function (RBF) and a multi layer perceptron (MLP) neural networks for the classification of breast abnormalities using texture features was performed by Christoyianni et al. [18] and Bovis et al. [16]. They concluded that MLP obtained 4\% higher accuracy than RBF. Yu et al. [31] used a multilayer feed forward neural network. They obtained good true positive accuracy at the cost of a very low false positive rate. Verma et al. [12] used a back-propagation neural network for the classification of suspicious lesions extracted using a fuzzy rule based detection system. They obtained $88.9 \%$ classification rate using a manual combination of features. Zhang et al. [13] used a genetic algorithm for neural network learning in their study of microcalcification pattern classification in digital mammograms. They have attained a good $90.5 \%$ accuracy rate on a test data set at the cost of a low accuracy rate on the training data set. Wroblewska et al. [32] proposed a new segmentation and feature extraction technique for the reliable classification of microcalcification patterns which achieved a low classification rate $(78 \%)$ on the DDSM database.

Despite the success of neural classifiers $[9-12,16,18,26]$ compared to other techniques in classifying breast abnormality patterns into benign and malignant classes, there are many drawbacks with current neural classifiers for the diagnosis of breast cancer. In other instances these problems are compounded due to malignant and benign abnormalities having similar characteristics [33]. Therefore, this paper focuses on removing some of these problems and improving the classification process. The primary aim of this study is to incorporate an unsupervised clustering algorithm such as selforganising map with a least square mechanism for determining clusters and weights of a multi-layer perceptron type neural network based classifier. The use of such a technique allows for the fast training of the classifier and overcomes the inherent problems of utilising clustering algorithms like backpropagation where a local minima or network paralysis could lead to less than optimal performance.

The remainder of this paper is organised into four sections. Section 2 discusses the proposed SOM-MGS approach. Section 3 presents the experimental results obtained with the proposed approach. Section 4 covers a brief discussion and a comparative analysis on the experimental results obtained with the proposed approach. In section 5, conclusions are drawn and future research directions are addressed.

\section{PROPOSED SOM-MGS APPROACH}

The proposed SOM-MGS approach is part of a full research methodology which is used to conduct experiments. The full research methodology is presented below in Figure 1 and all steps are described below in detail.

\section{A. Image Acquisition - Digital Mammogram}

The digital mammograms from the University of South Florida's Digital Database for Screening Mammography (DDSM) are used in this study. The DDSM is a benchmark database which is widely used by researchers to compare their research work with other researchers in the area of computer aided diagnosis of breast cancer [34]. The database contains approximately 2600 studies of malignant, benign, benignwithout-callback and normal cases and it has been divided into training and testing sets. The training set is used to train the system and the test set of data is used to test the system.

\section{B. Area Extraction}

The process of dividing a mammogram into distinct and discrete regions is called Area Extraction or Image Segmentation. This process is useful for subsequent phases since the mammogram is divided into Regions of Interest (ROI) which reduces the usage of system resources by discarding inappropriate regions. ROI represent regions of suspicion; however these regions will contain both malignant and benign abnormalities.

\section{Feature Extraction/Selection}

The characteristics (features) used to classify benign or malignant patterns can impact strongly on the systems performance and accuracy. Various features when utilised may be appropriate for classifying microcalcification anomalies but yield less than satisfactory results on mass type anomalies. It has therefore been argued that feature extraction and or selection could be the most important step in the process. Often a set of features are combined or used together since the classification accuracy of a single feature is not sufficiently discriminating in itself. When choosing a feature set the aim is to maximise the classification rate. A set of six features has been utilised in this research representing four BI-RADS descriptor features together with 
patient age and a subtlety value feature [34]. All six features are:

- Density

- Calcification Type / Mass Shape,

- Calcification Distribution / Mass Margin,

- Abnormality Assessment Rank

- Patient Age

- Subtlety Value

The BI-RADS lesion description features were specified by an expert Radiologist according to the BI-RADS lexicon. The case information associated with each case also includes information such as patient age when cancer was diagnosed. The subtlety value is a measure of how difficult a lesion is to find. Age is important since it has a correlation to the likelihood of developing breast cancer.

The feature sets are the inputs of the multi-layer perceptron Neural Network, which was used for performing the classification as benign or malignant classes. The Neural Network consisted of only one hidden. The number of nodes in the neural network was adjusted in an attempt to achieve optimal classification accuracy.

\section{Learning Process}

The learning process is comprised of several steps. SOM is utilised to cluster the input features into a number of clusters. The learned cluster values then become the input weights which are assigned to the hidden layer (Fig. 1) of a multilayer perceptron type neural network. The input weights of the hidden layer represent a weighting between the input (the feature set used for classification purposes - designated by (a)) and the hidden units of the neural network (b). In our research only a single hidden layer was used but with a variable number of hidden units which can impact on the overall performance of the system. Different numbers of hidden units were used in order to obtain an optimal range.

The output layer of the neural network (c) is the classification of the suspicious region as either a benign or malignant class. In order to achieve this, the network utilises weights between the hidden layer and the output layer. These weights need to be calculated or learned by the system. Once the number of hidden units has been specified for the system we expose it to a training set of mammograms where the benign or malignant class is known and calculate the weights which are adjusted as the system learns by using a modified Gram-Schmidt method. Once the weights from the clustering and modified Gram-Schmidt have been calculated on a training dataset they are then utilised to classify a test dataset to determine how effective the neural network classifier is, after it has been trained.

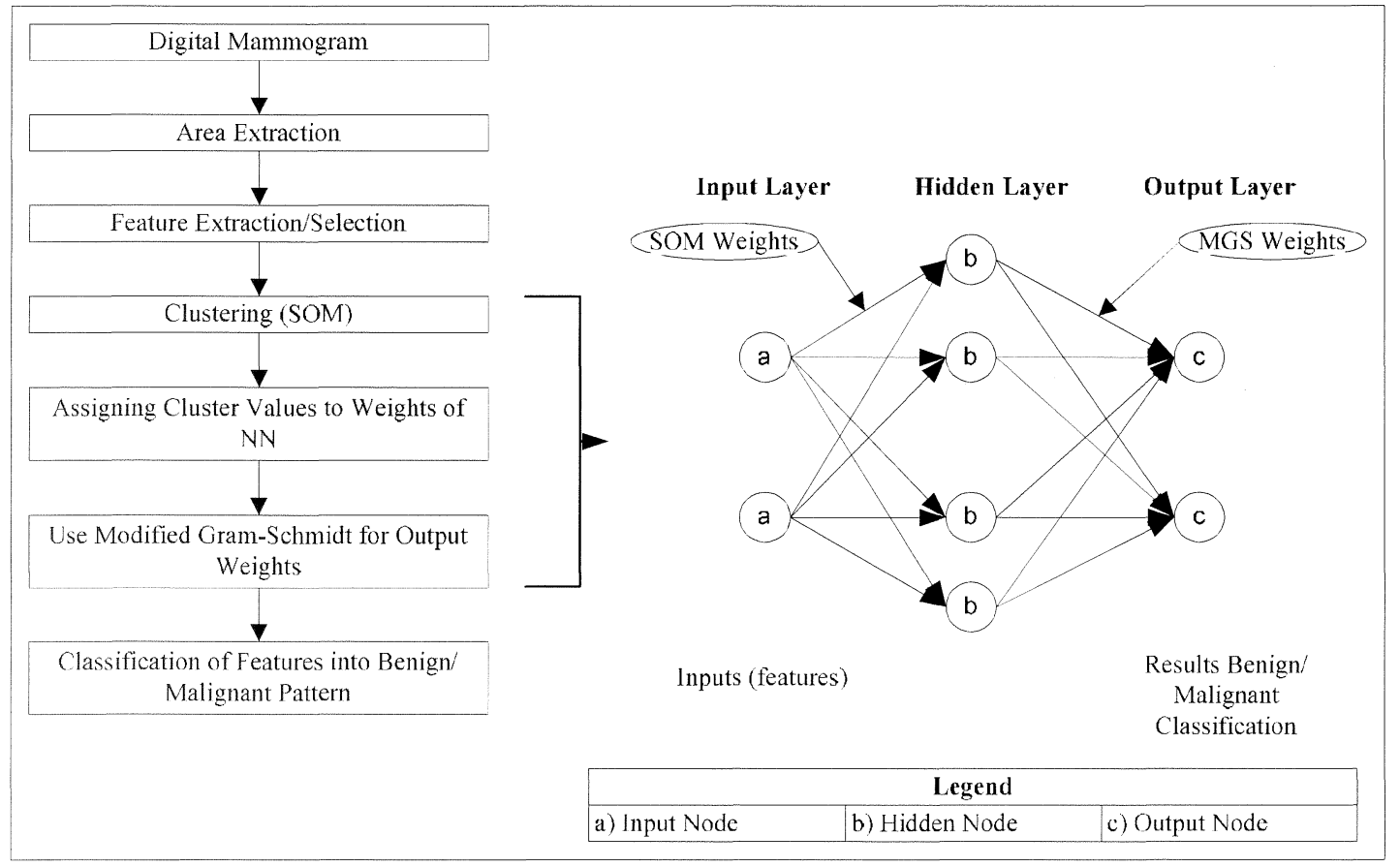

Fig. 1: An Overview of the Proposed Research Methodology 


\section{EXPERIMENTAL RESULTS}

The proposed approach has been implemented in $\mathrm{C}++$ on the Windows platform and is composed of two programs. The first performs the clustering using SOM and outputs the learned cluster weights to a file. The second program contains the neural network and reads the output cluster weights utilising this as the input weights to the neural network. The network is then trained to calculate the output weights utilising a modified gram-schmidt algorithm as detailed in the proposed SOM-MGS section of this paper. A number of experiments were performed by changing the number of clusters for SOM and number of hidden units (SOM-MGS). The features for the experiments were extracted from suspicious areas of digital mammograms taken from the DDSM database. The results for SOM-MGS and KmeansMGS (our previous research) are presented in Table 1.

\begin{tabular}{|c|c|c|c|c|}
\hline $\begin{array}{l}\text { No. of } \\
\text { Clusters }\end{array}$ & $\begin{array}{c}\text { No. of } \\
\text { Hidden Units }\end{array}$ & Research Methodology & \multicolumn{2}{|c|}{$\begin{array}{l}\text { Classification } \\
\text { Accuracy }[\%]\end{array}$} \\
\hline \multirow[t]{2}{*}{21} & \multirow[t]{2}{*}{19} & SOM-MGS & 95 & 92 \\
\hline & & Kmeans-MGS & 50 & 50 \\
\hline \multirow[t]{2}{*}{22} & \multirow[t]{2}{*}{19} & SOM-MGS & 94 & 88 \\
\hline & & Kmeans-MGS & 95 & 92 \\
\hline \multirow[t]{2}{*}{24} & \multirow[t]{2}{*}{24} & SOM-MGS & 94 & 91 \\
\hline & & Kmeans-MGS & 50 & 50 \\
\hline \multirow[t]{2}{*}{25} & \multirow[t]{2}{*}{21} & SOM-MGD & 96 & 85 \\
\hline & & Kmeans-MGS & 96 & 87 \\
\hline \multirow[t]{2}{*}{27} & \multirow[t]{2}{*}{17} & SOM-MGS & 94 & 86 \\
\hline & & Kmeans-MGS & 95 & 92 \\
\hline \multirow[t]{2}{*}{28} & \multirow[t]{2}{*}{28} & SOM-MGS & 95 & 87 \\
\hline & & Kmeans-MGs & 95 & 91 \\
\hline
\end{tabular}

The training and testing sets were each comprised of 100 mammograms. These sets each contained 50 malignant and 50 benign classes. The SOM clustering algorithm was run for 100, 200 and 1000 iterations. The SOM-MGS output weights were calculated for 2-40 hidden units and in all test cases the number of features was held constant at 6 .

The highest testing classification rate of $92 \%$ with the corresponding testing classification rate of $96 \%$ was attained with both SOM and Kmeans clustering algorithms. However the network topography that attained the highest testing classification rate of $92 \%$ with SOM-MGS couldn't work well with Kmeans-MGS

Both SOM-MGS and Kmeans-MGS obtained similar classification accuracy being $92 \%$ on the test data. However these results were obtained with a different number of clusters and nodes for each algorithm. The highest training classification rate was $96 \%$ for both clustering algorithms. It was however noted that the Kmeans-MGS tended to have a higher number of classifications for both the testing and training set at the highest classification accuracy in comparison to SOM-MGS. The number of iterations of the system did not impact on the results obtained. The higher consistent results obtained by using the k-means input weights indicates that it was better able to generalise in this instance.

\section{COMPARATIVE ANALYSIS}

The experiments presented in previous section were analysed and the results were compared with other existing approaches. The highest classification accuracy of $92 \%$ on test set was obtained. Figure 2 shows the classification rates attained by our proposed research methodology and existing techniques. Bovis et al. [16] attained $77 \%$ classification accuracy with BPNN and $78 \%$ with RBFNN on 161 breast images of MIAS dataset. Wu et al. [15] used a dataset containing 500 masses from the China Society for Industrial and Applied Mathematics. They reported highest $87.77 \%$ classification accuracy using the weighted average fusion algorithm on balanced input patterns to their $\mathrm{NN}$ ensembles and a highest $88.27 \%$ accuracy with the perceptron average fusion algorithm on imbalanced input patterns. Wroblewska et al. [32] have reported a $76 \%$ classification rate with their neural network based automated classification technique on the DDSM database. Panchal et al. [25] used an auto-associatorMLP based classifier and they reported $90.9 \%$ accuracy on test set; however the training of auto-associator and MLP took much longer time than the proposed methodology. Verma [35] proposed a neural algorithm which produced $94 \%$ accuracy on test set, however more iterations were used.

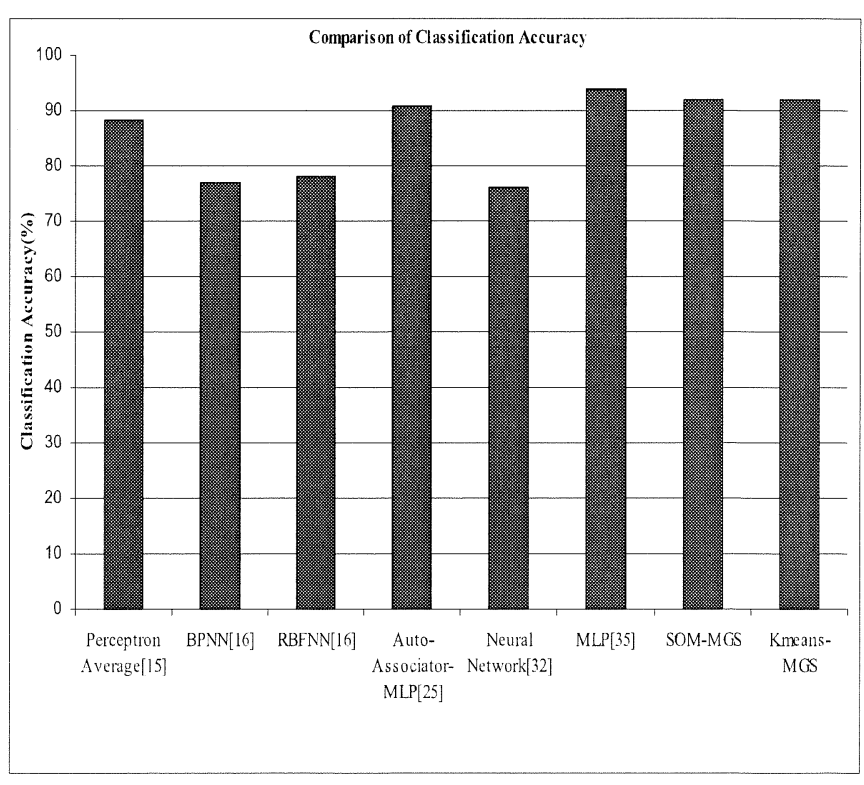

Fig. 2: Comparison of Classification Accuracy with Existing Techniques

\section{CONCLUSIONS}


We have presented a SOM-MGS based technique which has demonstrated that the combination of clustering algorithm such as SOM with modified Gram-Schmidt can produce good classification accuracy. However, more investigations with large number of clusters and other network parameters are needed to improve the $92 \%$ classification accuracy. The experiments showed that a small number of iterations and less training time in comparison to other techniques were required for SOM-MGS to obtain good clusters for hidden weights and overall training of the neural network. The modified GramSchmidt used for obtaining the output weights takes just one pass (non-iterative process) to adjust the weights. It was also noted that the k-means-MGS produced more consistent classification accuracy in comparison to SOM-MGS. The experiments performed were run for one feature set but varied the number of clusters and nodes. Our future research is to investigate more clusters and other feature sets to determine if a more optimal set of clusters and features will boost the classification accuracy of the system. Other research directions could also include alternative or multiple clustering algorithms.

\section{ACKNOWLEDGEMENT}

This work is supported by ARC ISSNIP Early Career Grants.

\section{REFERENCES}

[1] Breast Cancer Facts, 2005, www.breastcancerfund.org

[2] Pisano, E. D., Gatsonis, C., Hendrick, E., "Diagnostic Performance of Digital Versus Film Mammography for Breast Cancer Screening", New England Journal of Medicince, pp. 353, 2005.

[3] AbuBaker, A., Qahqaji, R., Aqel, M., Al-Osta, H. and Saleh, M., "Efficient Pre-processing of USF and MIAS Mammogram Images", Journal of Computer Science, Vol. 3, No. 2, pp. 67-75, 2006.

[4] Goergen, S., Evans, J., Cohen, G. and Macmillan, J., "Characteristics of Breast Carcinomas missed by Screening Radiologists", Radiology, Vol. 204, No. 11, pp. 131-135, 1997.

[5] Breast Cancer: www.wrongdiagnosis.com

[6] Birdwell, R. L., et al., "Mammographic Characteristics of 115 Missed Cancers Later Detected with Screening Mammography and the Potential Utility of Computer-aided Detection", Radiology, Vol. 219, pp. 192-202, 2001.

[7] Balleyguier, C., Kinkel, K., Fermanian, J., Malan, S., Djen, G., Taourel, P. and Helenon, O., "Computeraided detection (CAD) in mammography: Does it help the junior or the senior radiologist?", European Journal of Radiology, Vol. 54, pp. 90-96, 2005.

[8] Nodine, C., Kundel, H., Mello-Thoms, C., "How experience and training influence mammography expertise", Academic Radiology, Vol. 6, pp. 575-585, 1999.

[9] Cheng, H.D., Cai, X., Chen, X., Hu, L. and Lou, X., "Computer-aided Detection and Classification of Microcalcification in Mammograms: a survey", Pattern Recognition, Vol. 36, pp. 2967-2991, 2003.

[10] Cheng, H. D., Shi, X. J., Min, R., Ju, L. M., Cai, X. P. and $\mathrm{Du}, \mathrm{H}$. N., "Approaches for automated detection and classification of masses in mammograms", Pattern Recognition, Vol. 39, No. 4, pp. 646-68, 2006.

[11] Verma, B. and Panchal, R., "Neural Netowrks for the Classification of Benign Patterns in Digital Mammograms", Advances in Applied Artificial Intelligence, Idea Group, Inc., USA, Book Editor, John Fulcher, 2006.

[12] Verama, B. and Zakos, J., "A Computer-Aided Diagnosis System for Digital Mammograms Based on Fuzzy-Neural and Feature Extraction Techniques", IEEE Trans. On Info. Tech. in Biomedicine, Vol. 5, pp. 46-54, 2001

[13]Zhang, P., Verma, B. and Kumar, K., "A NeuralGenetic Algorithm for Feature Selection and Brest Abnormality Classification in Digital Mammography", Proc. of IEEE-IJCNN, Vol. 3, pp. 2303-2309, 2004.

[14]Zhang, P., Verma, B. and Kumar, K., "Neural Vs. Statistical Classifier in Conjunction with Genetic Algorithm Feature Selection in Digital Mammography", Pattern Recognition Letters, Vol. 26, pp. 909-919, 2005.

[15] Wu, U., He, J., Man, Y. and Arribas, J. I., "Neural Network Fusion Strategies for Identifying Breast Masses", Proc. of IEEE-IJCNN, Vol. 3, pp. 24372442, 2004.

[16] Bovis, K., Singh, S., Fieldsend, J. and Pinder, C., "Identification of Masses in Digital Mammograms with MLP and RBF Nets", Proc. of IEEE-IJCNN, Vol. 1, pp. 342-347, 2000.

[17] Chitre, Y., Dhawan, A. P. and Moskowitz, M., "Artificial Neural Netowrk Based Classification of Mammographic Microcalcifications using Image Structure Features", State of the Art in Digital Mammographic Image Analysis, World Scientific Publication, Vol. 9, pp. 167-197, 1994.

[18] Christoyianni, L., Dermatas, E. and Kokkinakis, G., "Neural Classification of Abnormal Tissues in Digital Mammography using Statistical Features of the Texture", Proc. of IEEE-ICECS, Vol. 1, pp. 117-120, 1999.

[19] Markey, M., Lo, J., Tourassi, G. and Floyd, C., "Selforganising Map for Cluster Analysis of a Breast Cancer Database", Artificial Intelligence in Medicine, Vol. 27, pp. 113-127, 2003

[20] Wu, Y., "Application of Neural Networks in Mammography”, Radiology, Vol. 187, pp. 81-87, 1993. 
[21] Guran, M., Chan, H., Sahiner, B., Hadjiiski, L., Petrick, N. and Helview, M., "Optimal Neural Network Architecture Selection: Improvement in Computerised Detection of Microcalcifications", Academic Radiology, Vol. 9, pp. 420-429, 2002.

[22] Papadopoulos, A., Fotiadis, D. I. and Likas, A., "An Automatic Microcalcification Detection System Based on a Hybrid Neural Network Classifier", Artificial Intelligence in Medicine, Vol. 25, pp. 149$167,2002$.

[23] Lo, J. Y., Land, W. H. and Morrison, C. T., "Application of Evolutionary Programming and Probabilistic Neural Networks to Breast Cancer Diagnosis", Proc. of IEEE IJCNN, Vol. 5, pp. $3712-$ $3716,1999$.

[24] Panchal, R. and Verma, B., "Neural Classification of Mass Abnormalities with Different types of Features in Digital Mammography, International Journal of Computational Intelligence and Applications", Vol. 6, pp. 61-67, 2006

[25] Panchal, R. and Verma, B., "Neural Association of Microcalcification Patterns for Their Reliable Classification in Digital Mammography", International Journal of Pattern Recognition and Artificial Intelligence, Vol. 20, pp. 971-983, 2006.

[26] Pena-Reyes, C. A. and Sipper, M., "A Fuzzy-Genetic Approach to Breast Cancer Diagnosis", Artificial Intelligence in Medicine, Vol. 17, pp. 131-155, 1999.

[27] Cheng, H., Lui, Y. M. and Freimanis, R. I., "A Novel Approach to Microcalcification Detection using Fuzzy Logic Technique", IEEE Trans. On Medical Imaging, Vol. 17, pp. 442-450, 1998.

[28] Lee, Y. and Tsai, D., "Computerized Classification of Microcalcifications on Mammograms using Fuzzy Logic and Genetic Algorithm", Medical Imaging, Proc. of SPIE, Vol. 5370, pp. 952-959, 2004.

[29] Yoshida, H., Nishikawa, R. N., Geiger, M. L. and Doi. K., "Signal/Background Separation By Wavelet Packets for Detection of Microcalcifications in Mammograms", Proc. of SPIE on Wavelet Applications in Signal and Image Processing IV, Vol. 2825 , pp. 805-811, 1996

[30] Wang, T. C. and Karayiannis, N. B., "Detection of Microcalcification in Digital Mammograms using Eavelets", IEEE Transactions on Medical Imaging, Vol. 17, pp. 498-509, 1998.

[31] Yu, S. and Guan, L., "A CAD System for the Automatic Detection of Clustered Microcalcifications in Digitized Mammogram Films", IEEE Trans. on Medical Imaging, Vol. 19, pp. 115-126, 2000.

[32] Wroblewska, A., Boninksi, P., Przelaskowski, A. and Kazubek, M., "Segmentation and feature extraction for reliable classification of microcalcification in digital mammograms", Opto-Electronics Review, Vol. 11, pp. 227-235, 2003.

[33]Zaiane, O., Antonie, M. and Coman, A. "Mammography Classification by an Association
Rule-based Classifier", Proc. of International Workshop on Multimedia Data Mining, 2002.

[34] Heath, M., Bowyer, K., Kopans, D., Moore, R. and KegelmeyerJr. P., "The Digital Database for Screening Mammography", IWDM-2000, Medical Physics Publishing, 2001.

[35] Verma, B. A Neural Learning Algorithm for the Diagnosis of Breast Cancer, IEEE International Joint Conference on Neural Networks, IJCNN'06, pp. 10786-10791, Canada, IEEE Press, 2006. 